\title{
A Narrative Review of update in per oral endoscopic myotomy (POEM) and endoscopic esophageal surgery
}

\author{
Antony Delliturri, Ory Wiesel, Jason Shaw, Igor Brichkov \\ Department of Surgery, Maimonides Medical Center, Brooklyn, NY, USA \\ Contributions: (I) Conception and design: I Brichkov; (II) Administrative support: O Wiesel; (III) Provision of study materials or patients: All authors; \\ (IV) Collection and assembly of data: All authors; (V) Data analysis and interpretation: All authors; (VI) Manuscript writing: All authors; (VII) Final \\ approval of manuscript: All authors. \\ Correspondence to: Antony Delliturri. Department of Surgery, Maimonides Medical Center, Brooklyn, New York, USA. Email: delliturria2@gmail.com.
}

\begin{abstract}
The field of endoscopic esophageal surgery is based on the concept of natural orifice transluminal endoscopic surgery (NOTES). Submucosal space surgery or third space surgery with the use of flexible endoscopy allows for decreased morbidity and hospital length of stay with equivalent outcomes for patients. In the case of achalasia, per oral endoscopic myotomy (POEM) allows for management of refractory cases in setting of previous Heller Myotomy or in patients whom laparoscopic or thoracoscopic surgery is contraindicated. Lastly, POEM more directly divides circular muscular layer of esophagus without destroying surrounding structures that exist to prevent reflux. The innovations in endoscopic surgery began in the animal lab with experiments in the porcine model to develop a way to access the peritoneal cavity through an entry point in the gastric mucosa. Over the last 10 years, the biggest treatment innovations in endoscopy have focused on management of achalasia with the use of POEM. POEM became possible as technology was developed that revolutionized the use of flexible technology and the methods of mucosal closure. In addition to benign esophageal disease, endoscopic methods improved in management of esophageal malignancy with endoscopic mucosal resection (EMR) or endoscopic submucosal dissection (ESD). The innovations of endoscopic surgery have been developed through the utilization of the submucosal space as a method to ensure adequate closure of the mucosal entry point into the peritoneal cavity. The goal of this review paper is to explore POEM and other techniques in endoscopic esophageal surgery for the management of esophageal diverticulum, submucosal tumors, gastroparesis, and gastrointestinal esophageal reflux disease.
\end{abstract}

Keywords: Endoscopic esophageal surgery; achalasia; per oral endoscopic myotomy (POEM); submucosal space surgery; natural orifice transluminal endoscopic surgery (NOTES)

Submitted Jul 01, 2020. Accepted for publication Jan 08, 2021.

doi: $10.21037 /$ atm-20-5057

View this article at: http://dx.doi.org/10.21037/atm-20-5057

\section{Introduction}

The field of endoscopic esophageal surgery is based on the concept of natural orifice transluminal endoscopic surgery (NOTES). NOTES was first described in the animal model by Kalloo et al., who performed a trans-gastric endoscopic liver biopsy (1). Worldwide acceptance was delayed due to limitations of endoscopic technology to allow for safe entry into the peritoneal cavity. Sumiyama et al. described the first animal experiments by creating a mucosal entry point into the submucosal space and creating an opening into the peritoneal cavity remote from the mucosal opening, allowing for a valve like closure (2). The concept of submucosal space surgery or "third space" surgery was invented through these experiments and facilitated through advancement in technology.

The worldwide acceptance of submucosal space surgery hinged on the development of POEM as the myotomy technique for achalasia. Following the success of endoscopic balloon dissection by Pasricha et al. in the porcine model, 
Inoue et al. published the first case series in 17 patients who underwent peroral endoscopic myotomy (POEM) (3). As in the porcine model, Inoue $e t$ al. created a mucosal entry site $10 \mathrm{~cm}$ proximal to the gastroesophageal junction (GEJ), and subsequent myotomy, while using a cautery device through the endoscope. The mucosal entry site was then closed via endoscopic clips. The results of the study showed significant reduction in lower esophageal sphincter (LES) pressure and associated reduction in dysphagia symptoms. They concluded that POEM is a safe and effective alternative to the Heller Myotomy for treatment of achalasia and should be accepted as standard of care (4).

The goal of submucosal space surgery with the use of flexible endoscopy is to reduce morbidity and decrease hospital length of stay without compromising outcomes for patients. Over the last ten years, the biggest treatment innovations in endoscopy have focused on management of achalasia with the use of POEM, and treatment of esophageal malignancy with endoscopic mucosal resection (EMR) or endoscopic submucosal dissection (ESD). The goal of this review is to explore POEM and other techniques in endoscopic esophageal surgery for the management of esophageal diverticulum, submucosal tumors, gastroparesis, and gastrointestinal esophageal reflux disease.

We present the following article in accordance with the Narrative Review reporting checklist (available at http:// dx.doi.org/10.21037/atm-20-5057).

\section{Achalasia}

Achalasia, despite being a rare esophageal motility disorder, effects about 6 in 100,000 persons per year worldwide. The majority of patients effected by achalasia are young women. The pathology is postulated to be secondary to destruction of nerves of the LES and degeneration of the neuromuscular function of the esophageal body to progressive pressurization, dilation, and loss of peristalsis (4). Achalasia requires diagnosis with initial barium esophagram and esophagoscopy to rule out disorders that present with similar symptoms like esophageal diverticula, malignancy and hiatal hernia $(3,4)$. Diagnosis is made with esophageal manometry and is defined through the Chicago Classification. The definition of classic achalasia based on manometry is aperistalsis of esophageal body with associated LES failure of relaxation and elevated integrated relaxation pressure (IRP) greater than $15 \mathrm{mmHg}$. The Chicago Classification system further characterizes the types of achalasia into three categories. Type 1 represents "classic achalasia" with esophageal aperistalsis and elevated IRP. Type 2 achalasia has elevated IRP, aperistalsis and esophageal body pressurization within normal caliber esophagus. Lastly, type 3 represents "spastic achalasia" with manometry findings of elevated IRP, esophageal aperistalsis, and simultaneous contractions of esophageal body (5).

\section{POEM}

Over the last decade, POEM has been shown to be an effective alternative to laparoscopic Heller myotomy (LHM) for the treatment of achalasia, as shown through a growing body of literature and world-wide acceptance. In 2019, there were over 109 articles available via PubMed on POEM with a total of 769 articles available since 2010 (6).

At our institution, POEM is performed by thoracic surgeons in the operating room via similar techniques described by Inoue et al. (4). The procedure is performed under general anesthesia in the supine position. A $6 \mathrm{~mm}$ channel gastroscope with a clear cap is used to allow for visualization during dissection. Cautery is delivered through the $6 \mathrm{~mm}$ channel via a triangle tip monopolar knife. Carbon dioxide is used for insufflation of the submucosal tunnel. Dissection is typically created in the space between the 2-o'clock and 5-o'clock position in the esophagus and is carried down to the lesser curve of the stomach. Maintaining dissection within this plane allows for preservation of the angle of His.

The procedure begins with initial esophagoscopy to establish the location of GEJ. The surgeon injects blue dye for the creation of the submucosal space. The mucosal opening is then created with a triangle tip knife proximal to the GEJ depending on the type of achalasia as described in the Chicago Classification. Longer myotomy length is typically required for type 2 and 3 achalasia with myotomy length up to $25 \mathrm{~cm}$. This submucosal tunnel extends distally to approximately $3 \mathrm{~cm}$ onto the gastric cardia. Following creation of the submucosal tunnel, endoscopic partial thickness myotomy of the circular muscles are created $5 \mathrm{~cm}$ distal to the initial mucosal opening, and extends to about $3 \mathrm{~cm}$ onto the stomach. The endoscope is then withdrawn from the submucosal tunnel and used to assess the effect of the myotomy. The mucosal opening is then closed with endoscopically placed clips. Prior to initiating a clear liquid diet, the myotomy is tested via gastrografin esophagram. Typically, our patients are discharged on the first postoperative day and instructed to continue a clear liquid diet for the first week. 


\section{POEM outcomes}

The therapeutic efficacy of POEM has been established by several groups with efficacy ranging from $82-98 \%$. The average time of follow-up in these studies ranged from 3 months to 3 years (5-10). Study design included multicenter retrospective review, case series, and meta-analysis. In a 2015 study by Inoue et al., 500 patients with achalasia underwent POEM at 1 institution between 2008 and 2013 with mean follow-up time of 3 years. They found a statistically significant reduction in the Eckhardt score from 6 to 1 . Despite success in treating achalasia, they found that $21.3 \%$ of patients at 3 years had gastroesophageal reflux disease (GERD) (7). A smaller case series performed by Shiwaku et al. found a similar reduction in the Eckhardt score from 5.9 to 0.8 after the POEM procedure. They found a $9 \%$ incidence of GERD at 3 months follow-up (8). Akintoye et al. evaluated 36 studies with 2,373 patients in a meta-analysis. Clinical success defined by Eckhardt score $<3$ was found in $98 \%$ of patients following POEM. The study reviewed adverse events including incidence of GERD, esophagitis, and abnormal acid exposure on ambulatory $\mathrm{pH}$ testing in $8.5 \%, 13 \%$, and $47 \%$, respectively (9). In a prospective, international, multi-center study conducted by Von Rentein et al., they looked at 70 patients over the course of 12 months. They found achalasia symptom remission in $82 \%$ of patients at 1 year (10). In a recent multi-center retrospective evaluation of 1,300 patients, clinical response was found in $94.7 \%$ of patients, and $14.8 \%$ had GERD at 1 year (11). The sum total of research on POEM suggests safety and efficacy for the treatment of achalasia at short- and long-term follow-up. Adverse events most commonly included GERD, which was treated with proton-pump inhibitors (9-11). Due to the desire to make POEM the standard of care for achalasia, there has been a push within the medical community to create novel techniques for the endoscopic management of GERD.

\section{Endoscopic management of GERD}

POEM associated GERD has influenced a rapidly growing body of research to create an endoscopic treatment option that can be performed either simultaneously with POEM, or as a separate procedure. Proponents of POEM envision a complete endoscopic treatment algorithm, that allows for the replacement of the LHM with partial fundoplication. Before discussion of ways to treat GERD, it is important to note that despite creation of a partial fundoplication during Heller myotomy, there is still evidence that GERD is present in $21-41.7 \%$ of patients $(12-14)$. The incidence of post-POEM GERD rates is $8.5-21 \%$, as discussed in the previous section (7-11). Treatment for GERD after POEM is typically managed with a PPI and endoscopic surveillance. The only endoscopic technique that exists for management of post-POEM GERD is POEM + fundoplication. Other novel endoscopic techniques have been developed, including the use of the Stretta device for radiofrequency ablation of the GEJ, Esophyx device for transoral incisionless fundoplication (TIF), and ARMS procedure for management of GERD.

The Stretta device uses radiofrequency energy at the GEJ and the proximal stomach via endoscopy. The goal is to create scar tissue that will ultimately act as reflux barrier, without causing an anatomic obstruction. Aziz et al. published a randomized control trial, using a double blinded sham model, that compared single dose and double dose radiofrequency ablation to a sham procedure. At 12 months follow-up, there was a $50 \%$ reduction in the double dose group, and $16.6 \%$ reduction in the single dose group. In addition, the sham procedure had no reduction in PPI use (15). In a long-term study by Noar et al. preformed over 10 years, they found reduction in symptoms associated with GERD. One hundred and fortynine patients were evaluated at 10 years with $64 \%$ having greater than $50 \%$ reduction in PPI, and $41 \%$ having complete discontinuation. In addition, they found that $51 \%$ of patients had no endoscopic esophagitis at 10 years.

Another novel device for endoscopic treatment of GERD was The Esophyx device, which is a novel approach to creating a reflux barrier by preforming an endoluminal fundoplication. Through this technique, a $2-3 \mathrm{~cm}$ 270-degree fundoplication is created at the GE junction. The TEMPO trial looked at 60 patients via randomized control trial comparing Esophyx TIF verses PPI. At 6 months, $97 \%$ of patients undergoing TIF versus $50 \%$ of PPI group had elimination of reflux symptoms. In addition, $90 \%$ of patients in TIF group had complete resolution of esophagitis, verses $38 \%$ in PPI group (16). Evidence for use of Esophyx device is promising, however, there is no evidence to compare this endoscopic procedure to the laparoscopic partial fundoplication. In addition, long-term data needs to be collected to further evaluate durability of the Esophyx device in preventing recurrence of GERD.

The most cost-effective approach to management of GERD is called anti-reflux mucosectomy (ARMS). The technique was described in a pilot study by Inoue et al 
in 2014 as an accidental finding following the treatment of Barrett's esophagus for high grade dysplasia. They observed that patients treated for Barrett's esophagus had improvement in GERD through the creation of a circumferential mucosal resection. The pilot study had 10 patients with GERD who underwent ARMS, which resulted in a statistically significant drop in the DeMeester score from 2.7 to $0.3(\mathrm{P}=0.0011)$ (17). In a larger prospective study, 62 patients were followed for 1 year and $72.5 \%$ $(\mathrm{P}<0.001)$ showed normalization of the DeMeester score. In this study, complications included dysphagia secondary to stricture in $8 \%$ of patients, which was treated with a single session of endoscopic dilation (18). The success and risk of complications of ARMS, based on the shortterm prospective data is low, however, long term outcomes will need to be established. ARMS should be considered for patients with refractory post-POEM GERD despite maximal Proton pump Inhibitor therapy.

The most innovative and novel approach to endoscopic fundoplication is a natural evolution of POEM by Inoue, who utilizes the submucosal space to access the peritoneal cavity and anchor the fundus of the stomach to the proximal esophagus. Inoue et al. recently published a pilot study preforming his novel endoscopic fundoplication that he called "POEM + F", using 21 patients. They concluded, based on this study, that POEM + F can serve as an alternative to the laparoscopic Heller-Dor procedure (19). Despite establishing feasibility of the procedure, further research is required to establish long-term data on outcomes in regard to reduction of GERD. The theoretical benefit of this procedure is the ability to perform the POEM and endoscopic fundoplication during the same procedure, without having the added costs of using the Stretta or Esophyx device $(20,21)$.

\section{Z-POEM}

Zenker diverticulum, first described in 1877 , is a false diverticulum that occurs in an area of natural weakness between the thyropharyngeus muscle and the cricopharyngeus muscle, within Killian's triangle $(22,23)$. The etiology is likely related to cricopharyngeus muscle dysfunction. Treatment is directed toward correcting the underlying sphincter dysfunction and managing the diverticulum (24). An endoscopic approach is preferred over the surgical treatment, as the former is associated with fewer complications, shorter procedure duration, and shorter length of stay in the hospital $(22,24)$. Endoscopic diverticulectomy requires division of the cricopharyngeus and the common wall between the esophagus and diverticulum, a technique popularized by Dohlman $(23,25)$. Recurrence of the diverticulum during traditional endoscopic technique is $11 \%$ based on the meta-analysis performed by Albers et al. (26). In 2016, Li et al. described a novel technique utilizing endoscopic submucosal tunneling and division of the muscular septum for treatment of Zenker's diverticulum, while preserving the mucosa through the use of a submucosal tunnel (27). Unlike the Dohlman technique where the diverticulum is divided with the mucosa, submucosa, and muscle layers, during the Z-POEM, a submucosal tunnel is created while maintaining an intact mucosal flap. In the meta-analysis by Ishaq et al., a total of 813 patients from 20 studies had a pooled success rate of $91 \%$, with a recurrence rate of $11 \%$. In addition, they found an adverse event rate of $11.3 \%$ (28). Another meta-analysis showed data from 589 patients in 13 studies with a success rate of $88 \%$, and complication rate of $13 \%$ (28). The use of submucosal space surgery to endoscopically divide the Zenker's diverticulum is a promising technique that will potentially become the standard of care; however, more research is required to compare the Z-POEM to the classic endoscopic treatment of Zenker's diverticulum in regard to durability, and safety.

\section{G-POEM}

The large success of POEM on the GEJ has resulted in the development of a separate procedure called gastric POEM (G-POEM). Like the POEM procedure, a mucosal incision is created proximal to the pylorus along the greater curvature of the stomach and the submucosal tunnel is created. The myotomy is performed at the pylorus and proximal stomach. Lastly, the mucosa is closed with endoscopic clips, thus completing the per oral pyloromyotomy (23). Typically, the procedure is performed only after other medical and interventional techniques have failed including botulinum toxin injection, stent placement, and the use of gastric stimulation device $(27,29,30)$. The pooled analysis of seven studies on G-POEM by Khoury et al. showed efficacy of $81.5 \%$, based on the Gastroparesis Cardinal Symptom Index, $7.6 \%$ perioperative complications, and $6.6 \%$ intraoperative complications (31). Rodriquez et al. published the largest study with 47 patients, which showed a $17 \%$ reduction of food retention at 4 hours $(\mathrm{P}<0.03)$ following G-POEM. Despite success with idiopathic gastroparesis, diabetic gastroparesis failed to show similar results in one study (32). An interesting consequence of G-POEM has been some 
reports of bile reflux gastritis (32), however, there is no randomized control study to support these events. Despite promising results on small retrospective studies, there still requires further follow up to determine long term success in alleviating gastroparesis symptoms, as compared to laparoscopic pyloromyotomy.

\section{EFTR and STER}

EMR and ESD have previously been the only methods of removing benign and malignant tumors from the gastrointestinal tract. These techniques are limited to tumors arising from the mucosa or submucosa. For lesions arising from muscularis propria, two novel alternatives exist including endoscopic full thickness resection (EFTR), and submucosal tunneling endoscopic resection (STER). EFTR is performed by completing full thickness resection of tumor, with subsequent endoscopic closure with clips. STER on the other hand capitalizes on a submucosal tunnel for the dissection of a mass. EFTR is limited to the stomach and colon due to the risk of post-resection stricture. STER on the other hand does not cause significant stricture, which allows the procedure to be performed in the esophagus (33-35).

The application of EFTR and STER include resection of submucosal tumors, including gastrointestinal stromal tumors, leiomyomas, schwannoma, granular cell tumor, lipoma, hemangioma, lymphangioma, fibroid tumor, lymphoma, neuroendocrine tumor, melanoma, and duplication cysts (35). Patients undergoing STER have been shown to have a decreased length of hospital stay as compared to those undergoing EFTR and thoracoscopic enucleation. No differences were found between EFTR and STER in regard to tumor size or rate of complications (33). However, EFTR and STER are most successful when tumors are less than 3 to $4 \mathrm{~cm}$ (34). There are no clear indications for either EFTR or STER, however, use for these techniques include tumors arising from muscularis propria at difficult to assess locations like the GEJ, or gastric cardia.

In a retrospective review of EFTR from a single hospital out of Switzerland, they found that there was a $97 \%$ technical success rate in 60 patients studied. Of the 60 patients studied, $79 \%$ had an R0 resection. Adverse events included bleeding in $7 \%$ of patients. No incidence of secondary perforation occurred in this study (36). In another retrospective study using EFTR for duodenal lesions, there was an overall success rate of $85 \%$, with an R0 resection rate of $63.2 \%$. Minor bleeding was found in $15.8 \%$ of patients, however, there was no evidence of secondary perforation (37).

STER has also been shown to be effective in removing submucosal tumors, and in a meta-analysis by $\mathrm{Lv}$ et al., they found a $97.5 \%$ complete resection rate in 28 studies. Complications included pneumomediastinum in $14.8 \%$, pneumoperitoneum in $6.8 \%$, and perforation in $5.6 \%$ of patients (38). Additional research into the efficacy of STER, through a long-term retrospective study of 180 patients, found resection rates of $90.6 \%$ and a complication rate of $8.3 \%$ after 36 months follow-up. The median duration of hospitalization was 3.2 days. A median follow-up of 36 months found no patients with local recurrence or distant metastasis (39). As compared to EFTR and thoracoscopic resection of submucosal tumors, STER is equally effective and exhibits shorter hospital stay for small benign lesions $(39,40)$.

\section{Conclusions}

Utilizing the submucosal space is a novel way to perform esophageal surgery, and has been advancing over the last decade since the popularization of POEM for achalasia. New methods of performing endoscopy within the submucosal space, has allowed for development of procedures for the treatment of achalasia, Zenker's diverticulum, gastroparesis, and the resection of subepithelial tumors. As endoscopic surgery techniques evolve, and surgeons continue to refine their endoscopic skills, submucosal space surgery will soon become the standard of care for the management of a variety of esophageal pathology.

\section{Acknowledgments}

Funding: None.

\section{Footnote}

Provenance and Peer Review: This article was commissioned by the editorial office, Annals of Translational Medicine for the series "Innovations and Updates in Esophageal Surgery". The article has undergone external peer review

Reporting Checklist: The authors have completed the Narrative Review reporting checklist. Available at http:// dx.doi.org/10.21037/atm-20-5057

Conflicts of Interest: All authors have completed the ICMJE uniform disclosure form (available at http://dx.doi. 
org/10.21037/atm-20-5057). The series "Innovations and Updates in Esophageal Surgery" was commissioned by the editorial office without any funding or sponsorship. OW served as the unpaid Guest Editor of the series and serves as an unpaid editorial board member of Annals of Translational Medicine from Oct 2019 to Sep 2021. The authors have no other conflicts of interest to declare.

Ethical Statement: The authors are accountable for all aspects of the work in ensuring that questions related to the accuracy or integrity of any part of the work are appropriately investigated and resolved.

Open Access Statement: This is an Open Access article distributed in accordance with the Creative Commons Attribution-NonCommercial-NoDerivs 4.0 International License (CC BY-NC-ND 4.0), which permits the noncommercial replication and distribution of the article with the strict proviso that no changes or edits are made and the original work is properly cited (including links to both the formal publication through the relevant DOI and the license). See: https://creativecommons.org/licenses/by-nc-nd/4.0/.

\section{References}

1. Kalloo AN, Singh VK, Jagannath SB, et al. Flexible transgastric peritoneoscopy: a novel approach to diagnostic and therapeutic interventions. Gastrointest Endosc 2004;60:114-7.

2. Sumiyama K, Gostout CJ, Rajan E, et al. Submucosal endoscopy with mucosal flap safety valve. Gastrointest Endosc 2007;65:688-94.

3. Pasricha PJ, Hawari R, Ahmed I, et al. Submucosal endoscopic esophageal myotomy: a novel experimental approach for the treatment of achalasia. Endoscopy 2007;39:761-4.

4. Inoue H, Minami H, Kobayashi $\mathrm{Y}$, et al. Peroral endocopic myotomy (POEM) for esophageal achalasia. Endoscopy 2010; 42:265-71.

5. Kahrilas PJ, Bredenoord AJ, Fox M, et al. The Chicago Classification of esophageal motility disorders, v3.0. Neurogastroenterol Motil 2015;27:160-74.

6. Parikh MP, Gupta NM, Sanaka MR. Esophageal Third Space Endoscopy: Recent Advances. Curr Treat Options Gastroenterol 2019;17:63-75.

7. Inoue H, Sato H, Ikeda H, et al. Per-Oral Endoscopic Myotomy: A Series of 500 Patients. J Am Coll Surg 2015;221:256-64.
8. Shiwaku H, Inoue H, Yamashita K, et al. Peroral endoscopic myotomy for esophageal achalasia: outcomes of the first over 100 patients with short-term follow-up. Surg Endosc 2016;30:4817-26.

9. Akintoye E, Kumar N, Obaitan I, et al. Peroral endoscopic myotomy: a meta-analysis. Endoscopy 2016;48:1059-68.

10. Von Renteln D, Fuchs KH, Fockens P, et al. Peroral endoscopic myotomy for the treatment of achalasia: an international prospective multicenter study. Gastroenterology 2013;145:309-11.e1-3.

11. Shiwaku $H$, Inoue $H$, Onimaru $M$, et al. Multicenter collaborative retrospective evaluation of peroral endoscopic myotomy for esophageal achalasia: analysis of data from more than 1300 patients at eight facilities in Japan. Surg Endosc 2020;34:464-8.

12. Kumagai K, Kjellin A, Tsai JA, et al. Toupet versus Dor as a procedure to prevent reflux after cardiomyotomy for achalasia: results of a randomized clinical trial. Int J Surg 2014;12:673-80.

13. Rawlings A, Soper N, Oelschlager B, et al. Laparoscopic Dor versus Toupet fundoplication following Heller myotomy for achalasia: results of a multicenter, prospective, randomized-controlled trial. Surg Endosc 2012;26:18-26.

14. Nieponice A, Nachman F, Badaloni A, et al. The impact of flexible endoscopy in esophageal surgery. J Thorac Dis 2017;9:S681-8.

15. Aziz AM, El-Khayat HR, Sadek A, et al. A prospective randomized trial of sham, single-dose Stretta, and doubledose Stretta for the treatment of gastroesophageal reflux disease. Surg Endosc 2010;24:818-25.

16. Trad KS, Barnes WE, Simoni G, et al. Transoral incisionless fundoplication effective in eliminating GERD symptoms in partial responders to proton pump inhibitor therapy at 6 months: the TEMPO Randomized Clinical Trial. Surg Innov 2015;22:26-40.

17. Inoue $\mathrm{H}$, Ito $\mathrm{H}$, Ikeda $\mathrm{H}$, et al. Anti-reflux mucosectomy for gastroesophageal reflux disease in the absence of hiatus hernia: a pilot study. Ann Gastroenterol 2014;27:346-51.

18. Patil G, Dalal A, Maydeo A. Feasibility and outcomes of anti-reflux mucosectomy for proton pump inhibitor dependent gastroesophageal reflux disease: First Indian study (with video). Dig Endosc 2020;32:745-52.

19. Inoue H, Ueno A, Shimamura Y, et al. Peroral endoscopic myotomy and fundoplication: a novel NOTES procedure. Endoscopy 2019;51:161-4.

20. Ishaq S, Sultan H, Siau K, et al. New and emerging techniques for endoscopic treatment of Zenker's 
diverticulum: State-of-the-art review. Dig Endosc 2018;30:449-60.

21. Hernández Mondragón OV, Solórzano Pineda MO, Blancas Valencia JM. Zenker's diverticulum: Submucosal tunneling endoscopic septum division (Z-POEM). Dig Endosc 2018;30:124.

22. Brieau B, Leblanc S, Bordacahar B, et al. Submucosal tunneling endoscopic septum division for Zenker's diverticulum: a reproducible procedure for endoscopists who perform peroral endoscopic myotomy. Endoscopy 2017;49:613-4.

23. Zenker FA, von Ziemssen H. Handbuch der Krankheiten des Chylopoëtischen Apparates I. In: Krankenheiten des oesophagus. Leipzig: F.C.W. Vogel, 1877:1-87.

24. Li QL, Chen WF, Zhang XC, et al. Submucosal Tunneling Endoscopic Septum Division: A Novel Technique for Treating Zenker's Diverticulum. Gastroenterology 2016;151:1071-4.

25. Nabi Z, Nageshwar D, Ramchandani M. Recent Advances in Third-Space Endoscopy. Gastroenterol Hepatol (N Y) 2018;14:224-32.

26. Albers DV, Kondo A, Bernardo WM, et al. Endoscopic versus surgical approach in the treatment of Zenker's diverticulum: systematic review and meta-analysis. Endosc Int Open 2016;4:E678-86.

27. Li LY, Yang YT, Qu CM, et al. Endoscopic needleknife treatment for symptomatic esophageal Zenker's diverticulum: A meta-analysis and systematic review. J Dig Dis 2018;19:204-14.

28. Ishaq S, Hassan C, Antonello A, et al. Flexible endoscopic treatment for Zenker's diverticulum: a systematic review and meta-analysis. Gastrointest Endosc 2016;83:1076-89.e5.

29. Khashab MA, Stein E, Clarke JO, et al. Gastric peroral endoscopic myotomy for refractory gastroparesis: first human en- doscopic pyloromyotomy (with video). Gastrointest Endosc 2013;78:764-8.

30. Mekaroonkamol P, Dacha S, Wang L, et al. Gastric Peroral Endoscopic Pyloromyotomy Reduces Symptoms, Increases Quality of Life, and Reduces Health Care Use For Patients With Gastroparesis. Clin Gastroenterol

Cite this article as: Delliturri A, Wiesel O, Shaw J, Brichkov I. A Narrative Review of update in per oral endoscopic myotomy (POEM) and endoscopic esophageal surgery. Ann Transl Med 2021;9(10):909. doi: 10.21037/atm-20-5057
Hepatol 2019;17:82-9.

31. Khoury T, Mizrahi M, Mahamid M, et al. State of the art review with literature summary on gastric peroral endoscopic pyloromyotomy for gastroparesis. J Gastroenterol Hepatol 2018;33:1829-33.

32. Rodriguez JH, Haskins IN, Strong AT, et al. Per oral endoscopic pyloromyotomy for refractory gastroparesis: initial results from a single institution. Surg Endosc 2017;31:5381-8.

33. Gonzalez JM, Benezech A, Vitton V, et al. G-POEM with antro-pyloromyotomy for the treatment of refractory gastroparesis: mid-term follow-up and factors predicting outcome. Aliment Pharmacol Ther 2017;46:364-70.

34. McCabe ME 4th, Dilly CK. New causes for the old problem of bile reflux gastritis. Clin Gastroenterol Hepatol 2018;16:1389-92.

35. Dellatore P, Bhagat V, Kahaleh M. Endoscopic full thickness resection versus submucosal tunneling endoscopic resection for removal of submucosal tumors: a review article. Transl Gastroenterol Hepatol 2019;4:45.

36. Manolakis AC, Inoue H, Ueno A, et al. 2007-2019: a "Third"-Space Odyssey in the Endoscopic Management of Gastrointestinal Tract Diseases. Curr Treat Options Gastroenterol 2019;17:202-20.

37. Nishida T, Kawai N, Yamaguchi S, et al. Submucosal tumors: comprehensive guide for the diagnosis and therapy of gastrointestinal submucosal tumors. Dig Endosc 2013;25:479-89.

38. Lv XH, Wang CH, Xie Y. Efficacy and safety of submucosal tunneling endoscopic resection for upper gastrointestinal submucosal tumors: a systematic review and meta-analysis. Surg Endosc 2017;31:49-63.

39. Valli PV, Mertens J, Bauerfeind P. Safe and successful resection of difficult GI lesions using a novel single-step full-thickness resection device (FTRD $((\mathrm{R})))$. Surg Endosc 2018;32:289-99.

40. Bauder M, Schmidt A, Caca K. Endoscopic full-thickness resection of duodenal lesions-a retrospective analysis of 20 FTRD cases. United European Gastroenterol J 2018;6:1015-21. 